\title{
Rational-Harmonic Balancing Approach to Nonlinear Phenomena Governed by Pendulum-Like Differential Equations
}

\author{
Encarnación Gimeno and Augusto Beléndez \\ Departamento de Física, Ingeniería de Sistemas y Teoría de la Señal, Universidad de Alicante, \\ Apartado 99, E-03080 Alicante, Spain \\ Reprint requests to A. B.; Fax: +34-6-5909750; E-mail: a.belendez@ua.es
}

Z. Naturforsch. 64a, 1 - 8 (2009); received October 6, 2008 / February 9, 2009

\begin{abstract}
This paper presents a new approach for solving accurate approximate analytical solutions for nonlinear phenomena governed by pendulum-like differential equations. The new approach couples Taylor series expansion with rational harmonic balancing. An approximate rational solution depending on a small parameter is considered. After substituting the approximate solution into the governing differential equation, this equation is expanded in Taylor series of the parameter prior to harmonic balancing. The approach gives a cubic equation, which must be solved in order to obtain the value of the small parameter. A method for transforming this cubic equation into a linear equation is presented and discussed. Using this approach, accurate approximate analytical expressions for period and periodic solutions are obtained. We also compared the Fourier series expansions of the analytical approximate solution and the exact one. This allowed us to compare the coefficients for the different harmonic terms in these solutions. These analytical approximations may be of interest for those researchers working in nonlinear physical phenomena governed by pendulum-like differential equations in fields such as classical mechanics, vibrations, acoustics, electromagnetism, electronics, superconductivity, optics, gravitation, and others.
\end{abstract}

Key words: Nonlinear Oscillator; Rational Harmonic Balance Method; Approximate Solutions; Nonlinear Pendulum.

\section{Introduction}

Nonlinear oscillations in physics, mathematics, and engineering has been a topic to intensive research for many years. A large variety of approximate technologies have been developed to determine the periodic solutions of nonlinear oscillatory systems. The most common and most widely studied methods of all approximation methods for nonlinear differential equations are perturbation methods [1,2]. Some other techniques include variational iteration [3-6], Exp-function [7], homotopy perturbation [8-12], equivalent linearization [13], standard and modified Lindstedt-Poincaré $[14,15]$, parameter expanding [15-17], harmonic balance $[1,18,19]$ methods, etc. Surveys of the literature with numerous references, useful bibliography, and a review of these methods can be found in detail in [4] and [15].

The simple pendulum oscillatory motion is among the most investigated motions in physics $[2,11,19,20-24]$ and many nonlinear phenomena in real world are governed by pendulum-like differential equations [21,22]. As Lima [22] pointed out, these nonlinear equations arise in many fields of science and technology, such as acoustic vibrations [2], oscillations in small molecules [21], oscillations of buildings during earthquakes [25], post-buckling in cantilever columns [26], optically torqued nanorods [27], Josephson superconductivity junctions [21,28], elliptic filters for electronic devices [21], analysis of smectic $C$ liquid crystals [29], gravitational lensing in general relativity [30], advanced models in field theory [31], and others.

The method of harmonic balance is a wellestablished procedure for determining analytical approximations to the solutions of differential equations, the time domain response of which can be expressed as Fourier series [1]. In the usual harmonic balance methods, the solution of a nonlinear system is assumed to be of the form of a truncated Fourier series [1, 18]. Being different from the other nonlinear analytical methods, such as perturbation techniques, the harmonic balance method does not depend on small parameters, such that it can find wide application in nonlinear problems without linearization or small perturbations. Various generalizations of the harmonic balance method have 
been made and one of them is the rational representation proposed by Mickens [1,32-34]. In this method the approximate solution obtained approximates all of the harmonics in the exact solution [34,35], whereas the usual harmonic balance techniques provide an approximation to only the lowest harmonic components. For most cases, the application of the rational harmonic balance method leads to very complicated sets of algebraic equations with very complex nonlinearity that have to be solved even for the second-order approximation. In an attempt to provide better solution methodology a modification in this technique is proposed for constructing the second-order analytical approximate solution. In this paper a modified rational harmonic balance method is investigated in terms of the truncation terms of the series expansion of the nonlinear differential equations. This new procedure has been proved to be effective for various conservative nonlinear oscillations with odd nonlinearities $[36,37]$ and now it is proposed for constructing approximate analytical periodic solutions for nonlinear phenomena governed by pendulum-like differential equations.

\section{Solution Procedure}

The dimensionless nonlinear differential equation for the free, undamped simple pendulum is

$$
\frac{\mathrm{d}^{2} x}{\mathrm{~d} t^{2}}+\sin x=0
$$

with the initial conditions

$$
x(0)=A \text { and } \frac{\mathrm{d} x}{\mathrm{~d} t}(0)=0 .
$$

The periodic solution $x(t)$ of (1) and the period depend on the amplitude $A$. In the small-angle regime, the approximation $\sin x \approx x$ works, yielding the usual linearization for (1), as given by

$$
\frac{\mathrm{d}^{2} x}{\mathrm{~d} t^{2}}+x=0 .
$$

For the initial conditions given in (2), the solution of this problem is the well-known expression $x(t)=$ $A \cos t$ and in this regime the motion is harmonic with a dimensionless frequency $\omega=1$ and a period $T=2 \pi$. Beyond this regime, (1) can be numerically solved but we are interested in analytical approximations.

There exists no small parameter in (1), so the standard perturbation methods cannot be applied directly.
Due to the fact that the rational harmonic balance method (RHBM) requires neither a small parameter nor a linear term in a differential equation [1], one possibility to approximately solve (1) is by means of this method. The main purpose of this paper is to construct an analytical approximation to the solution of (1) using a modified RHBM introduced by Beléndez et al. $[36,37]$ and which has been applied for truly conservative nonlinear oscillators with good results. To solve (1) by the modified RHBM, a new independent variable $\tau=\omega t$ is introduced. Then (1) and (2) can be rewritten as

$$
\begin{aligned}
& \omega^{2} \frac{\mathrm{d}^{2} x(\tau)}{\mathrm{d} \tau^{2}}+\sin (x(\tau))=0, \\
& x(0)=A, \quad \frac{\mathrm{d} x}{\mathrm{~d} \tau}(0)=0 .
\end{aligned}
$$

The new independent variable is chosen in such a way that the solution of (4) is a periodic function of $\tau$ of pe$\operatorname{riod} 2 \pi$. The corresponding frequency of the nonlinear oscillator is $\omega$ and it is a function of the amplitude $A$.

In order to determine an improved approximation we use a rational form given by the following expression [1]:

$$
x_{2}(\tau)=\frac{A_{1} \cos \tau}{1+B_{2} \cos 2 \tau} .
$$

In this equation it is satisfied that $\left|B_{2}\right| \ll 1$, and $A_{1}$ and $B_{2}$ are constants, one of which can be determined from the initial conditions expressed in (5), which yield $A_{1}=A\left(1+B_{2}\right)$, i. e.,

$$
x_{2}(\tau)=\frac{\left(1+B_{2}\right) A \cos \tau}{1+B_{2} \cos 2 \tau},
$$

whose substitution into (4) yields

$$
\begin{aligned}
& -\omega^{2} \frac{A\left(1+B_{2}\right) \cos \tau}{1+B_{2} \cos 2 \tau}+\omega^{2} \frac{4 A B_{2}\left(1+B_{2}\right) \cos 3 \tau}{\left(1+B_{2} \cos 2 \tau\right)^{2}} \\
& +\omega^{2} \frac{8 A B_{2}^{2}\left(1+B_{2}\right) \cos \tau \sin ^{2} 2 \tau}{\left(1+B_{2} \cos 2 \tau\right)^{3}} \\
& +\sin \left(\frac{A\left(1+B_{2}\right) \cos \tau}{1+B_{2} \cos 2 \tau}\right) \approx 0
\end{aligned}
$$

which can be written as follows:

$$
F\left(A, B_{2}, \omega, \tau\right)=0 .
$$

As $\left|B_{2}\right| \ll 1$ we can do the following Taylor series expansion:

$$
\begin{aligned}
F\left(A, B_{2}, \omega, \tau\right) \approx & F_{0}(A, \omega, \tau)+F_{1}(A, \omega, \tau) B_{2} \\
& +F_{2}(A, \omega, \tau) B_{2}^{2},
\end{aligned}
$$


where

$$
\begin{aligned}
F_{0}(A, \omega, \tau)=-A \omega^{2} \cos \tau+\sin (A \cos \tau), & \\
F_{1}(A, \omega, \tau)= & -\frac{1}{2} \omega^{2} A(\cos \tau-9 \cos 3 \tau) \\
& +\frac{1}{2} A(\cos \tau-\cos 3 t) \cos (A \cos \tau), \\
F_{2}(A, \omega, \tau)= & \frac{1}{4} \omega^{2} A(9 \cos 3 \tau-25 \cos 5 \tau) \\
& -A(\cos \tau+\cos 3 \tau) \sin ^{2} \tau \cos (A \cos \tau) \\
& -2 A^{2} \cos ^{2} \tau \sin ^{4} \tau \sin (A \cos \tau) .
\end{aligned}
$$

Expanding (10) in a trigonometric serie and taking into account (9) we can write

$$
\begin{aligned}
& H^{(1)}\left(A, B_{2}, \omega\right) \cos \tau+H^{(3)}\left(A, B_{2}, \omega\right) \cos 3 \tau \\
& \quad+\mathrm{HOH}=0,
\end{aligned}
$$

where $\mathrm{HOH}$ stands for higher-order harmonics and

$$
\begin{aligned}
H^{(1)}\left(A, B_{2}, \omega\right)= & f_{0}^{(1)}(A, \omega)+f_{1}^{(1)}(A, \omega) B_{2} \\
& +f_{2}^{(1)}(A, \omega) B_{2}^{2}, \\
H^{(3)}\left(A, B_{2}, \omega\right)= & f_{0}^{(3)}(A, \omega)+f_{1}^{(3)}(A, \omega) B_{2} \\
& +f_{2}^{(3)}(A, \omega) B_{2}^{2} .
\end{aligned}
$$

In (15) and $(16), f_{m}^{(1)}(A, \omega)$ and $f_{m}^{(3)}(A, \omega)(m=0,1,2)$ are given as follows:

$$
\begin{aligned}
f_{m}^{(1)}(A, \omega) & =\frac{4}{\pi} \int_{0}^{\pi / 2} F_{m}(A, \omega, \tau) \cos \tau \mathrm{d} \tau \\
f_{m}^{(3)}(A, \omega) & =\frac{4}{\pi} \int_{0}^{\pi / 2} F_{m}(A, \omega, \tau) \cos 3 \tau \mathrm{d} \tau
\end{aligned}
$$

and their analytical expressions are

$$
\begin{aligned}
f_{0}^{(1)}(A, \omega)= & -\omega^{2} A+2 J_{1}(A), \\
f_{1}^{(1)}(A, \omega)= & -\frac{1}{2} \omega^{2} A+4 J_{1}(A)-\frac{12}{A} J_{2}(A), \\
f_{2}^{(1)}(A, \omega)= & \frac{8}{A^{3}}\left[\left(-30+A^{2}\right) A J_{1}(A)\right. \\
& \left.+3\left(40-3 A^{2}\right) J_{2}(A)\right], \\
f_{0}^{(3)}(A, \omega)= & 2 J_{3}(A)-8 \frac{J_{2}(A)}{A} \\
f_{1}^{(3)}(A, \omega)= & \frac{9}{2} \omega^{2} A+\left(4-\frac{240}{A^{2}}\right) J_{1}(A) \\
& +\left(-\frac{60}{A}+\frac{960}{A^{3}}\right) J_{2}(A),
\end{aligned}
$$

$$
\begin{aligned}
f_{2}^{(3)}(A, \omega)= & \frac{9}{4} \omega^{2} A+\left(8-\frac{2640}{A^{2}}+\frac{60480}{A^{4}}\right) J_{1}(A) \\
& -\left(\frac{264}{A}-\frac{20640}{A^{3}}+\frac{241920}{A^{5}}\right) J_{2}(A) .
\end{aligned}
$$

In (19)-(24), $J_{n}(A)$ is the $n$-order Bessel function of the first kind.

Setting the coefficients of $\cos \tau$ and $\cos 3 \tau$ to zero in (14) it follows that

$$
\begin{aligned}
& H^{(1)}\left(A, B_{2}, \omega\right)=0, \\
& H^{(3)}\left(A, B_{2}, \omega\right)=0,
\end{aligned}
$$

which allow us to obtain $B_{2}$ and the second-order approximate frequency $\omega$ as a function of $A$. Solving (25) for $\omega$ yields

$$
\begin{aligned}
\omega(A)= & \frac{2}{A^{2} \sqrt{2+B_{2}}}\left\{\left[A^{3}+2 A^{3} B_{2}\right.\right. \\
& \left.+\left(4 A^{3}-120 A\right) B_{2}^{2}\right] J_{1}(A) \\
& \left.-\left[6 A^{2} B_{2}+6\left(6 A^{2}-80\right) B_{2}^{2}\right] J_{2}(A)\right\}^{1 / 2}
\end{aligned}
$$

and substituting (27) into (26) gives the following cubic equation which must be solved to obtain $B_{2}$ :

$$
B_{2}^{3}+a_{2}(A) B_{2}^{2}+a_{1}(A) B_{2}+a_{0}(A)=0,
$$

where

$$
\begin{aligned}
& a_{2}(A)=\frac{b_{2}(A)}{b_{3}(A)}, \\
& a_{1}(A)=\frac{b_{1}(A)}{b_{3}(A)}, \\
& a_{0}(A)=\frac{b_{0}(A)}{b_{3}(A)},
\end{aligned}
$$

and $b_{0}, b_{1}, b_{2}$, and $b_{3}$ are given as follows:

$$
\begin{aligned}
b_{0}= & 2 A^{4} J_{1}(A)-8 A^{3} J_{2}(A), \\
b_{1}= & \left(-240 A^{2}+13 A^{4}\right) J_{1}(A) \\
& +\left(960 A-60 A^{3}\right) J_{2}(A), \\
b_{2}= & \left(60480-2640 A^{2}+26 A^{4}\right) J_{1}(A), \\
- & \left(\frac{241920}{A}-20640 A+318 A^{3}\right) J_{2}(A), \\
b_{3}(A)= & \left(-1080 A^{2}+36 A^{4}\right) J_{1}(A) \\
+ & \left(4320 A-324 A^{3}\right) J_{2}(A) .
\end{aligned}
$$


The polynomial discriminant $D(A)$ of the cubic equation given in (28) is defined as follows [1]:

$$
D(A)=\frac{p^{3}(A)}{27}+\frac{q^{2}(A)}{4}
$$

where

$$
\begin{aligned}
& p(A)=\frac{3 a_{1}(A)-a_{2}^{2}(A)}{3}, \\
& q(A)=\frac{9 a_{1}(A) a_{2}(A)-27 a_{0}(A)-2 a_{2}^{3}(A)}{27} .
\end{aligned}
$$

Determining which roots of (28) are real and which are complex can be accomplished by noting that, if the polynomial discriminant $D>0$, one root is real and two are complex conjugates; if $D=0$, all roots are real and at least two are equal; and if $D<0$, all roots are real and unequal [1]. As we can see (28) is cubic and therefore has three solutions. However, the root of interest is one for which $\left|B_{2}\right| \ll 1$. To proceed, assume that such a solution $B_{2}$ exists and its value is lower than one. A first approximate value $B_{2}^{(1)}$ can be determined by neglecting the first two terms in (28)

$$
a_{1}(A) B_{2}^{(1)}+a_{0}(A)=0 .
$$

Solving this equation gives

$$
B_{2}^{(1)}(A)=-\frac{a_{0}(A)}{a_{1}(A)} .
$$

To further improve this result, we assume that $B_{2}$ can be written as follows:

$$
B_{2}=B_{2}^{(1)}+\delta \text {, }
$$

where $\delta$ is a correction term and $|\delta| \ll\left|B_{2}^{(1)}\right|$. Substituting (41) into (28) and linearizing with respect to the correction term $\delta$ lead to

$$
\begin{aligned}
& {\left[B_{2}^{(1)}\right]^{3}\left(1+\frac{3 \delta}{B_{2}^{(1)}}\right)+a_{2}\left[B_{2}^{(1)}\right]^{2}\left(1+\frac{2 \delta}{B_{2}^{(1)}}\right)} \\
& +a_{1} B_{2}^{(1)}\left(1+\frac{\delta}{B_{2}^{(1)}}\right)+a_{0}=0,
\end{aligned}
$$

which is a linear equation instead of a cubic equation. Solving (42) it follows that

$$
\begin{aligned}
B_{2} & =-\frac{a_{0}\left(2 a_{0}^{2}+a_{1}^{3}-a_{0} a_{1} a_{2}\right)}{a_{1}\left(3 a_{0}^{2}+a_{1}^{3}-2 a_{0} a_{1} a_{2}\right)} \\
& =-\frac{b_{0}\left(2 b_{0}^{2} b_{3}+b_{1}^{3}-b_{0} b_{1} b_{2}\right)}{b_{1}\left(3 b_{0}^{2} b_{3}+b_{1}^{3}-2 b_{0} b_{1} b_{2}\right)},
\end{aligned}
$$

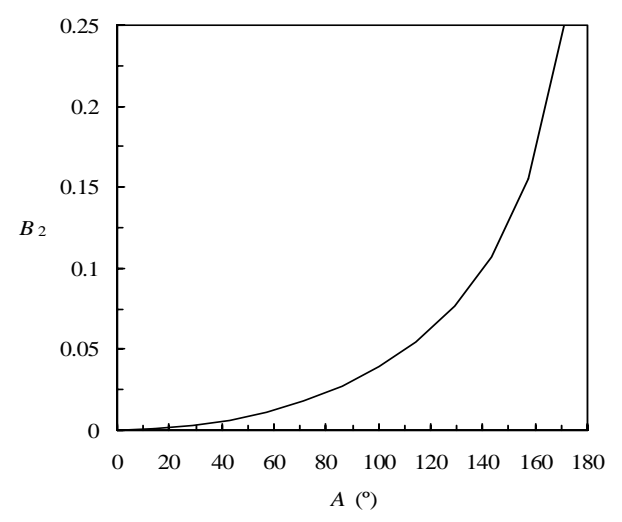

Fig. 1. Parameter $B_{2}$ as a function of $A$.

where (43) has been taken into account and $b_{0}, b_{1}, b_{2}$, and $b_{3}$ are given in (32)-(35). In Figure 1 we have plotted $B_{2}$ as a function of $A$.

Substituting (43) into (27) we obtain the value for the second-order approximate frequency $\omega_{2}$ as a function of $A$. Therefore, the second approximation to the periodic solution of the nonlinear oscillator is given by the following equation:

$$
\frac{x_{2}(t)}{A}=\frac{\left(1+B_{2}\right) \cos \omega_{2} t}{1+B_{2} \cos 2 \omega_{2} t} \text {. }
$$

This periodic solution has the following Fourier series expansion:

$$
\frac{x_{2}(t)}{A}=\sum_{n=0}^{\infty} a_{2 n+1} \cos \left[(2 n+1) \omega_{2} t\right],
$$

where

$a_{2 n+1}=\frac{4}{\pi} \int_{0}^{\pi / 2} \frac{\left(1+B_{2}\right) \cos \tau}{1+B_{2} \cos 2 \tau} \cos [(2 n+1) \tau] \mathrm{d} \tau$.

As we can see, (44) gives an expression that approximates all of the harmonics in the exact solution, whereas the usual harmonic balancing techniques provide and approximate only the lowest harmonic components.

\section{Results and Discussion}

We illustrate the accuracy of the modified approach by comparing the approximate solutions previously obtained with the exact period $T_{\mathrm{ex}}=2 \pi / \omega_{\mathrm{ex}}$ for the nonlinear pendulum. The exact value of the period of oscillations is given by the equation [38]

$$
T_{\mathrm{ex}}=T_{0} \frac{2}{\pi} K(k),
$$


where $k=\sin ^{2}(A / 2)$ and $K(k)$ is the complete elliptic integral of the first kind defined as follows:

$$
K(k)=\int_{0}^{\pi / 2} \frac{\mathrm{d} \theta}{\sqrt{1-k \sin ^{2} \theta}} .
$$

For small values of $A$ it is possible to do the power series expansions of the exact and approximate angular periods $T_{\mathrm{ex}}(A)$ (see $(47)$ ) and $T_{2}(A)=2 \pi / \omega_{2}(A)$ (see (27)). Doing these expansions, the following equations can be obtained:

$$
\begin{aligned}
\frac{T_{\mathrm{ex}}}{2 \pi}= & 1+\frac{1}{16} A^{2}+\frac{11}{3072} A^{4}+\frac{173}{737280} A^{6} \\
& +\frac{22931}{1321205760} A^{8}+\ldots, \\
\frac{T_{2}}{2 \pi}= & 1+\frac{1}{16} A^{2}+\frac{11}{3072} A^{4}+\frac{175}{737280} A^{6} \\
& +\frac{23583}{1321205760} A^{8}+\ldots
\end{aligned}
$$

Taking into account (32) - (35), we can also obtain the power series expansion of $B_{2}$ (see (43)) for small values of $A$ and we obtain

$$
B_{2}=\frac{1}{96} A^{2}+\frac{11}{18432} A^{4}+\frac{43}{1105920} A^{6}+\ldots
$$

These series expansions were carried out using MATHEMATICA. As can be seen in (50), the first three terms are the same as the first three terms obtained from the expansion of the exact period $T_{\mathrm{ex}}$ (see (49)), whereas the fourth term of the expansion of the exact period is $\frac{173}{737280}$ compared with $\frac{175}{737280}$ obtained in our study, that is, the relative error in this term is $1.2 \%$. The fifth term in the expansion of the exact period is $\frac{22931}{1321205760} \mathrm{com}-$ pared with $\frac{23583}{1321205760}$ obtained in this study, that is, the relative error in this term is $2.8 \%$. As we can see the second-order approximate period $T_{2}(A)=2 \pi / \omega_{2}(A)$ obtained in this paper provides excellent approximations to the exact period $T_{\mathrm{ex}}(A)$ for high values of the oscillation amplitude $A$.

Comparison of the exact period $T_{\mathrm{ex}}$ obtained using (47), with the proposed period $T_{2}$ computed using (27) and (43) is shown in Table 1 for different values of the initial amplitude $A$. The percentage errors have been computed by using the following equation:

$$
P E(\%)=100\left|\frac{T_{2}-T_{\mathrm{ex}}}{T_{\mathrm{ex}}}\right| .
$$

Table 1. Comparison of the second-order approximate period obtained using the rational harmonic balance method $\left(T_{2}\right)$ with the exact one $\left(T_{\mathrm{ex}}\right)$ and relative errors. In the first column the values of $B_{2}$ obtained using (43) are included.

\begin{tabular}{rlccl}
\hline$A\left(^{\circ}\right)$ & $B_{2}$ & $T_{2}$ & $T_{\mathrm{ex}}$ & $P E(\%)$ \\
\hline 20 & 0.0012782 & 6.33137 & 6.33137 & 0.0000005 \\
40 & 0.0052233 & 6.48011 & 6.48010 & 0.000033 \\
60 & 0.012197 & 6.74303 & 6.74300 & 0.00041 \\
80 & 0.022913 & 7.14726 & 7.14708 & 0.0026 \\
100 & 0.038691 & 7.74323 & 7.74232 & 0.012 \\
120 & 0.062088 & 8.62993 & 8.62606 & 0.045 \\
140 & 0.098824 & 10.0349 & 10.0182 & 0.17 \\
160 & 0.16679 & 12.7121 & 12.6135 & 0.78 \\
170 & 0.23687 & 15.7113 & 15.3270 & 2.5 \\
\hline
\end{tabular}

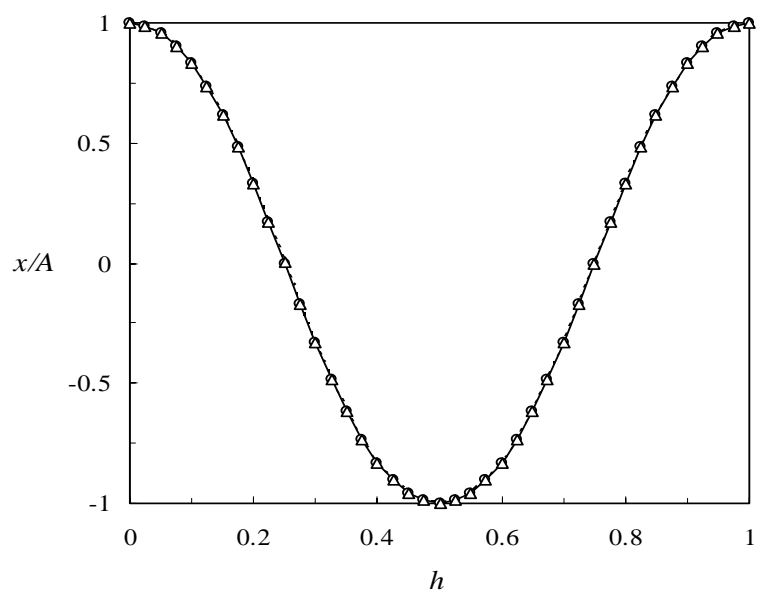

Fig. 2. Comparison of the normalized second-order approximate solution ( $\triangle$ and dashed line) with the exact solution ( $\circ$ and continuous line) for $A=100^{\circ}$.

For the rational harmonic approximation constructed in this paper, the relative error between the approximate and the exact values of the period is less than $1 \%$ for $A<162.5^{\circ}$.

The normalized exact periodic solution [38]:

$$
\begin{aligned}
\frac{x_{\mathrm{ex}}(t)}{A}= & \frac{2}{A} \arcsin \left\{\sin \left(\frac{A}{2}\right)\right. \\
& \left.\cdot \operatorname{sn}\left[K\left(\sin ^{2}\left(\frac{A}{2}\right)\right)-t ; \sin ^{2}\left(\frac{A}{2}\right)\right]\right\},
\end{aligned}
$$

where $\operatorname{sn}(v ; m)$ is the Jacobi elliptic function. The approximate periodic solutions $x_{2} / A$ in (44), are plotted in Figures $2-5$ for $A=100^{\circ}, 120^{\circ}, 140^{\circ}$, and $160^{\circ}$, respectively. In these figures, the parameter $h$ is defined as $h=t / T_{\mathrm{ex}}(A)$. These figures show that (27), (43), and (44) can provide high accurate approximations to the exact frequency and the exact periodic solutions for $A<160^{\circ}$. 


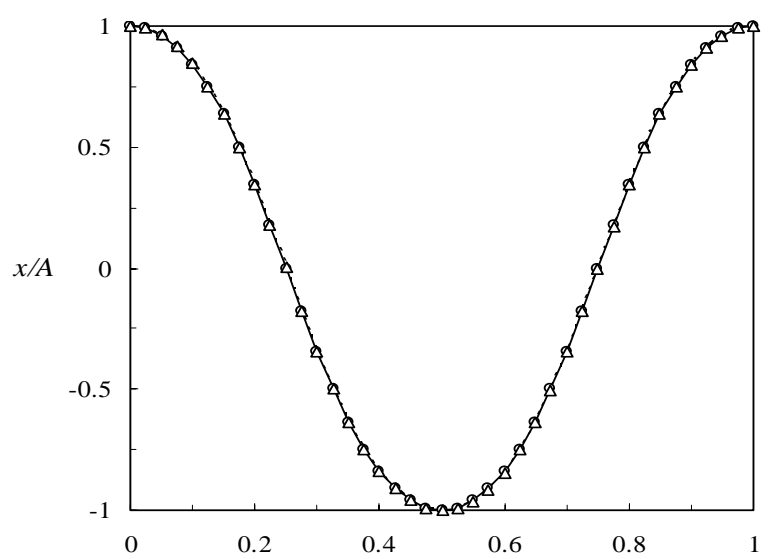

$h$

Fig. 3. Comparison of the normalized second-order approximate solution ( $\triangle$ and dashed line) with the exact solution (o and continuous line) for $A=120^{\circ}$.

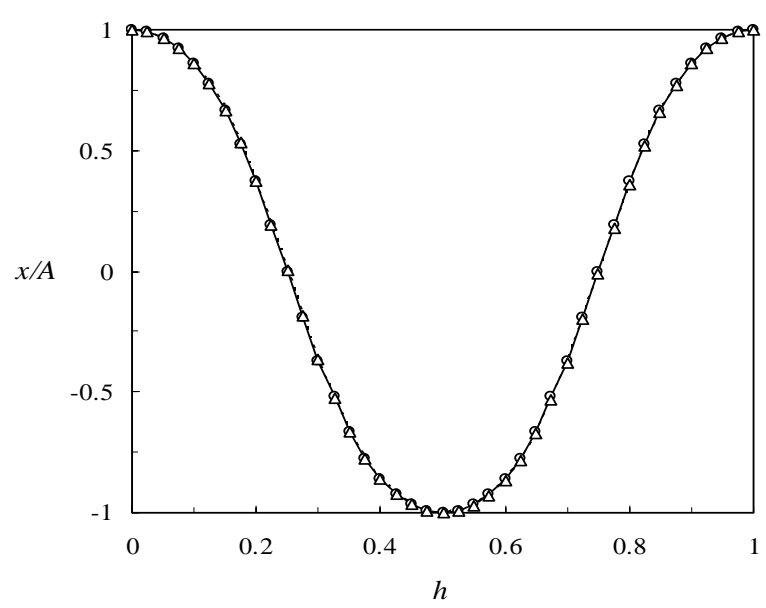

Fig. 4. Comparison of the normalized second-order approximate solution ( $\triangle$ and dashed line) with the exact solution (० and continuous line) for $A=140^{\circ}$.

The Fourier representation of (44) is given in (45). The following result was obtained by Beléndez et al. [36] for the Fourier coefficients $a_{2 n+1}$ :

$$
\begin{aligned}
a_{2 n+1}= & (-1)^{n} 2^{n+1} \sqrt{\frac{1+B_{2}}{1-B_{2}}}\left(\frac{B_{2}}{1-B_{2}}\right)^{n} \\
& \cdot\left(\frac{\sqrt{1-B_{2}}}{\sqrt{1-B_{2}}+\sqrt{1+B_{2}}}\right)^{2 n+1} .
\end{aligned}
$$

The Fourier expansion of (53) is

$$
\frac{x_{\mathrm{ex}}(t)}{A}=\sum_{n=0}^{\infty} b_{2 n+1} \cos \left[(2 n+1) \omega_{\mathrm{ex}} t\right],
$$

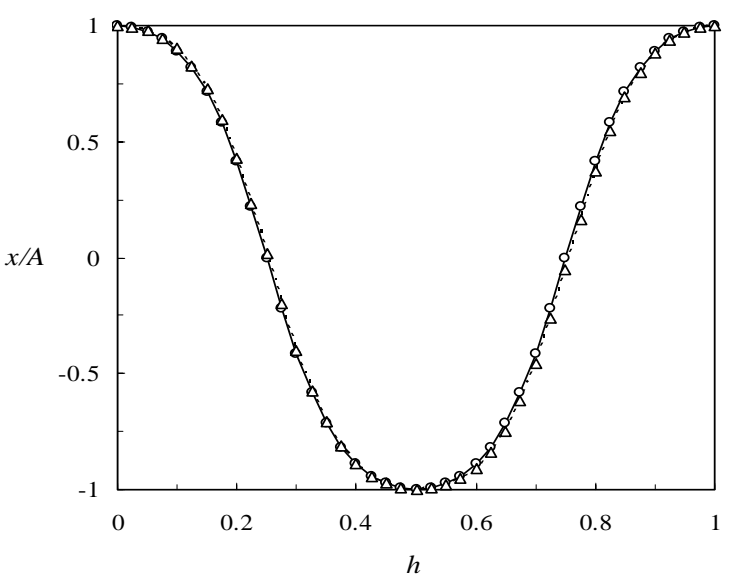

Fig. 5. Comparison of the normalized second-order approximate solution ( $\triangle$ and dashed line) with the exact solution (० and continuous line) for $A=160^{\circ}$.

where

$$
b_{2 n+1}=\frac{4}{\pi} \int_{0}^{\pi / 2} \frac{x_{\mathrm{ex}}(\eta)}{A} \cos [(2 n+1) \eta] \mathrm{d} \eta .
$$

From (53), (55), and (56) we obtain for $A=30^{\circ}$ and $\eta=\omega_{e x} t$

$$
\begin{aligned}
\frac{x_{\mathrm{ex}}(t)}{A}= & 1.00145 \cos \omega_{\mathrm{ex}} t \\
& -0.00145284 \cos 3 \omega_{\mathrm{ex}} t \\
& +0.00000377745 \cos 5 \omega_{\mathrm{ex}} t-\ldots,
\end{aligned}
$$

while from (44) and (54), and also for $A=30^{\circ}$, it follows

$$
\begin{aligned}
\frac{x_{2}(t)}{A}= & 1.00145 \cos \omega_{2} t \\
& -0.00145284 \cos 3 \omega_{2} t \\
& +0.00000210768 \cos 5 \omega_{2} t-\ldots
\end{aligned}
$$

For $A=60^{\circ}$ we obtain

$$
\begin{aligned}
\frac{x_{\mathrm{ex}}(t)}{A}= & 1.00607 \cos \omega_{\mathrm{ex}} t \\
& -0.00613545 \cos 3 \omega_{\mathrm{ex}} t \\
& +0.0000661616 \cos 5 \omega_{\mathrm{ex}} t-\ldots, \\
\frac{x_{2}(t)}{A}= & 1.00610 \cos \omega_{2} t \\
& -0.00613573 \cos 3 \omega_{2} t \\
& +0.000037419 \cos 5 \omega_{2} t-\ldots
\end{aligned}
$$

For $A=90^{\circ}$ we obtain

$$
\begin{aligned}
\frac{x_{\mathrm{ex}}(t)}{A}= & 1.01487 \cos \omega_{\mathrm{ex}} t \\
& -0.0152493 \cos 3 \omega_{\mathrm{ex}} t \\
& +0.00039542 \cos 5 \omega_{\mathrm{ex}} t-\ldots,
\end{aligned}
$$




$$
\begin{aligned}
\frac{x_{2}(t)}{A}= & 1.01503 \cos \omega_{2} t \\
& -0.0152546 \cos 3 \omega_{2} t \\
& +0.000229256 \cos 5 \omega_{2} t-\ldots
\end{aligned}
$$

These equations allow us to compare the first Fourier coefficients of the Fourier series expansions of exact and analytical approximate solutions for different values of $A$.

\section{A Simpler Approximate Formulae for the Nonlinear Pendulum}

Now we can obtain two simple expressions for the periodic solutions and the frequency for which the relative error for the frequency is less than $1 \%$ for $A=$ $124^{\circ}$. For small values of $A$ we can approximate (51) as

$$
B_{2}(A) \approx \frac{1}{96} A^{2}+\frac{11}{18432} A^{4} .
$$

Substituting (63) into (27) and doing the power series expansion for small values of $A$, we obtain

$$
T_{2}(A)=\frac{2 \pi}{\omega_{2}(A)} \approx \frac{2 \pi}{\sqrt{1-\frac{1}{8} A^{2}+\frac{7}{1536} A^{4}}} .
$$

Substituting (63) and (64) into (7) leads to

$$
\begin{aligned}
& x_{2}(t) \approx \\
& \frac{\left(A+\frac{1}{96} A^{3}+\frac{11}{18432} A^{5}\right) \cos \left(\sqrt{1-\frac{1}{8} A^{2}+\frac{7}{1536} A^{4} t}\right)}{1+\left(\frac{1}{96} A^{2}+\frac{11}{18432} A^{4}\right) \cos \left(2 \sqrt{1-\frac{1}{8} A^{2}+\frac{7}{1536} A^{4} t}\right)} .
\end{aligned}
$$

[1] R.E. Mickens, Oscillations in Planar Dynamics Systems, World Scientific, Singapore 1996.

[2] P. Amore and A. Aranda, J. Sound Vib. 283, 1115 (2005).

[3] J.H. He, Chaos, Solitons \& Fractals 34, 1430 (2007).

[4] J. H. He, Int. J. Mod. Phys. B 223487 (2008).

[5] E. M. Abulwafa, M. A. Abdou, and A.H. Mahmoud, Z. Naturforsch. 63a, 131 (2008).

[6] S. Abbasbandy and E. Shivanian, Z. Naturforsch. 63a, 538 (2008).

[7] F. Xu, Z. Naturforsch. 62a, 685 (2007).

[8] M. T. Darvishi and F. Khani, Z. Naturforsch. 63a, 19 (2008).

[9] J. H. He, Int. J. Nonlinear Sci. Numer. Simul. 6, 207 (2005).

\section{Conclusions}

Based on the rational harmonic balance method a new procedure has been developed and has been used to determine analytical approximate solutions for nonlinear phenomena governed by pendulum-like differential equations. This method is based on the substitution of a rational solution, depending on a small parameter $B_{2}$, into the nonlinear differential equation, Taylor series expansion with respect to the small parameter $B_{2}$, neglect of the terms proportional to $B_{2}^{n}(n \geq 3)$ in the Taylor series expansion, and harmonic balancing. The major conclusion is that this scheme provides excellent approximations to the solution of these nonlinear systems with high accuracy. The relative error of the second-order analytical approximate frequency obtained using the approach considered in this paper is lower than $1 \%$ for oscillation amplitudes as high as $162^{\circ}$. In summary, this new procedure to apply the rational harmonic balance method is very simple in its principle, and it can be used to solve other conservative nonlinear oscillators.

\section{Acknowledgements}

This work was supported by the "Generalitat Valenciana" of Spain under project ACOMP/2009/150 and by the "Vicerrectorado de Tecnologa e Innovación Educativa" of the University of Alicante, Spain (GITE09006-UA).

[10] A. Beléndez, M. L. Alvarez, D. I. Méndez, E. Fernández, M. S. Yebra, and T. Beléndez, Z. Naturforsch. 63a, 529 (2008).

[11] A. Beléndez, A. Hernández, T. Beléndez, C. Neipp, and A. Márquez, Eur. J. Phys. 28, 93 (2007).

[12] M. M. Mousa and S. F. Ragab, Z. Naturforsch. 63a, 140 (2008).

[13] A. Beléndez, C. Pascual, C. Neipp, T. Beléndez, and A. Hernández, Int. J. Nonlinear Sci. Numer. Simul. 9, 9 (2008).

[14] J. H. He, Int. J. Nonlinear Mech. 37, 309 (2002).

[15] J. H. He, Int. J. Mod. Phys. B, 20,1141 (2006).

[16] L. N. Zhang and L. Xu, Z. Naturforsch. 62a, 396 (2007).

[17] M. T. Darvishi, A. Karami, and B. C. Shin, Phys. Lett. A 372, 538 (2008). 
[18] S. K. Lai, Y. Xiang, C.W. Lim, X.F. He, and Q.C. Zeng, J. Sound Vib. 317, 440 (2008).

[19] A. Beléndez, A. Hernández, A. Márquez, T. Beléndez, and C. Neipp, Eur. J. Phys. 27, 539 (2006).

[20] F. M. S. Lima and P. Arun, Am. J. Phys. 74, 892 (2006).

[21] G. L. Baker and J.A. Blackburn, The Pendulum: A Case Study in Physics, Oxford University Press, Oxford 2005 .

[22] F. M. S. Lima, Eur. J. Phys. 29, 1091 (2008).

[23] P. Amore, M.C. Valdovinos, G. Orneles, and S.Z. Barajas, Rev. Mex. Fis. E 53, 106 (2007).

[24] M. S. Alam, M. E. Haque, and M. B. Hossain, Int. J. Nonlinear Mech. 42, 1035 (2007).

[25] A.C. Lazer and P. J. McKenna, SIAM Rev. 32, 537 (1990).

[26] A. Valiente, Am. J. Phys. 72, 1008 (2004).

[27] W. A. Shelton, K. D. Bonin, and T. G. Walker, Phys. Rev. E 71, 036204 (2005).

[28] A. Marchenkov, R.W. Simmonds, J.C. Davis, and R. E. Packard, Phys. Rev. B 61, (2000) 4196.
[29] G. J. Barclay and I. W. Stewart, J. Phys. A: Math. Gen. 33, 4599 (2000).

[30] P. Amore, M. Cervantes, A. de Pace, and F.M. Fernández, Phys. Rev. D 75, 083005 (2007).

[31] V.E. Grishin and V. K. Fedyanin, Theor. Math. Phys. 59, 609 (1984)

[32] K. Cooper and R. E. Mickens, J. Sound. Vib. 250, 951 (2002).

[33] R. E. Mickens, J. Sound Vib. 111, 515 (1986).

[34] R. E. Mickens and D. Semwogerere, J. Sound Vib. 195, 528 (1996).

[35] A. Beléndez, E. Gimeno, M.L. Álvarez, S. Gallego, M. Ortuño, and D. I. Méndez, Int. J. Nonlinear Sci. Numer. Simul. 10, 57 (2009).

[36] A. Beléndez, E. Gimeno, E. Fernández, D. I. Méndez, and M. L. Alvarez, Phys. Scr. 77, 065004 (2008).

[37] A. Beléndez, E. Gimeno, M. L. Alvarez, D. I. Méndez, and A. Hernández, Phys. Lett. A 372, 6047 (2008).

[38] A. Beléndez, C. Pascual, D. I. Méndez, T. Beléndez, and C. Neipp, Rev. Bras. Ens. Fis. 29, 645 (2007). 